UVX 2010 (2011) 233-237

DOI: $10.1051 /$ uvx/2011035

(C) Owned by the authors, published by EDP Sciences, 2011

\title{
Femtosecond laser pulse absorption by dielectrics: Surface experiments and modelling
}

\author{
N. Varkentina ${ }^{1, *}$, O. Utéza ${ }^{1}$, B. Chimier ${ }^{1}$, N. Sanner ${ }^{1}$, M. Sentis ${ }^{1}$ and T. Itina ${ }^{2}$ \\ ${ }^{1}$ LP3, UMR 6182 CNRS - U. de la Méditerranée, 163 Av. de Luminy, c. 917, 13009 Marseille, \\ France \\ ${ }^{2}$ LAHC, UMR 5516 CNRS - U. J. Monnet, 18 rue Pr. B. Lauras - Bât. F, \\ 42000 Saint-Étienne, France
}

\begin{abstract}
Experimental and theoretical investigations are performed to measure and understand the physical mechanisms of laser energy absorption at the surface of a dielectric material excited by a femtosecond laser.
\end{abstract}

\section{INTRODUCTION}

We perform experiments and modelling to understand the femtosecond laser energy deposition at the surface of a dielectric material. The experiments consist of measuring the incident, reflected and transmitted beams as a function of fluence in order to retrieve the evolution of laser absorption in the material. The experiments are compared to a modelling simulating the evolution of ionization of the material as a function of time and magnitude of the energy deposition. Confrontation of experimental and modelling results allows clarifying the absorption mechanisms at the femtosecond timescale.

\section{EXPERIMENTS}

The set-up is shown in Fig. 1. A linearly polarized Yb:KGW laser (Amplitude Systèmes, S-pulse) provides $\tau=450 \mathrm{fs}$ pulses at $1025 \mathrm{~nm}$. The Pockels cell is used for picking single shot pulses from the $1 \mathrm{kHz}$ pulse train. The samples consist of Suprasil fused silica (Heraeus), with impurity $<0.065 \mathrm{ppm}$, residual roughness $\mathrm{Ra}=0.2 \mathrm{~nm}$ and bandgap $E_{\text {gap }}=9 \mathrm{eV}$. They are positioned at the focal plane of the incident beam focused with a $50 \mathrm{~mm}$ focal lens $\left(\omega_{0}=6.3 \mu \mathrm{m}\right)$. The energy $\mathrm{E}$ is adjusted by a computer controlled half-waveplate coupled to a Brewster polarizer. For each energy case (fluence F), the results are averaged on 20 shots, each one on a fresh site of the sample. To determine the amount of energy absorbed by the sample, we measure the reflected and transmitted pump signals by two photodiodes. Spectral and spatial filters are used for optical isolation of the signals with respect to the broadband plasma emission emitted on a wide solid angle. Afterwards, we suppose that: $R+T+A=$ 1 , with $R=R_{\text {spec }}+R_{\text {diff }}$ and $T=T_{\text {spec }}+T_{\text {diff }} . R_{\text {spec }}, T_{\text {spec }}$ and $R_{\text {diff }}, T_{\text {diff }}$ respectively indicate the contribution of the specular (measured in our experiment) and diffuse reflection and transmission. We neglect the changes in $R_{\text {diff }}$ and $T_{\text {diff }}$ as the pump duration is considerably smaller than the timescale on which the modification of the material properties yielding large variations of these parameters (morphology change) take place ( $\gg$ ps). Finally, as we are working with aninitial perfectly optical

\footnotetext{
*e-mail: varkentina@lp3.univ-mrs.fr

This is an Open Access article distributed under the terms of the Creative Commons Attribution-Noncommercial License 3.0, which permits unrestricted use, distribution, and reproduction in any noncommercial medium, provided the original work is properly cited.
} 
polished surface $\left(R_{\text {diff }} \ll R_{\text {spec }}, T_{\text {diff }} \ll T_{\text {spec }}\right)$, we assume: $A=E_{\text {abs }} / E_{\text {inc }}=1-R_{\text {spec }}-T_{\text {spec }}=$ $1-E_{\text {refl }} / E_{\text {inc }}-E_{\text {trans }} / E_{\text {inc }}$. Finally, we verify that the free electron population created following laser excitation of the sample does not significantly vary the beam polarization and thus influence the measured $\mathrm{R}$ and $\mathrm{T}$ signals. We then measure the two polarization state ( $\mathrm{s}$ and $\mathrm{p}$ ) components of the transmitted signal. The results show that the signal collected on the residual polarization state (here the s-component) is negligible with respect to the p-component (Fig. 2). So we can conclude that there is no plasma influence on the polarization state of the transmitted beam.

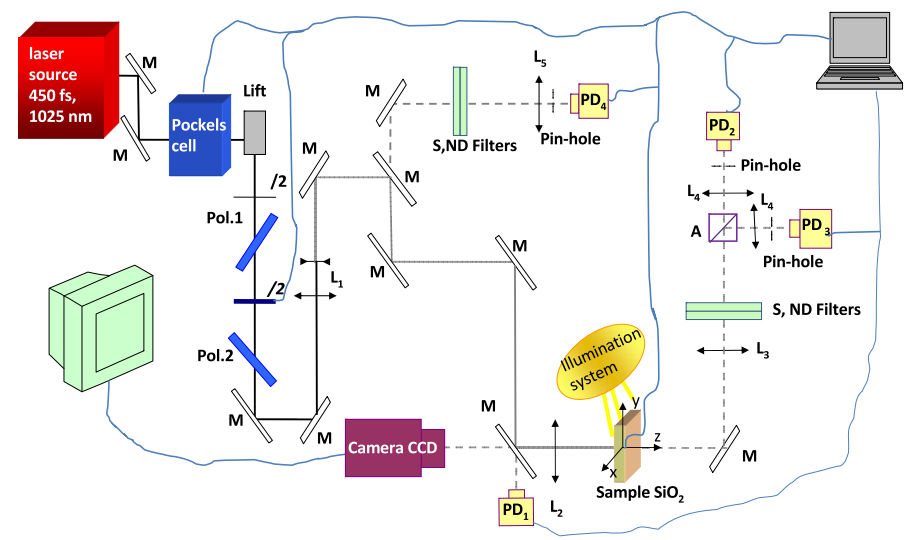

Figure 1. Experimental damaging and ablation test-bench and pump-pump reflection and transmission diagnostics. $\mathrm{M}$-mirror; Lift—beam elevating system; $\lambda / 2$-half-waveplate; Pol.1 and Pol.2-Brewster polarizers; $\mathrm{L}_{1}$ telescopic system; S, ND filters-spectral (longpass cut-on filter at $1000 \mathrm{~nm}$ combined to a Si-photodetector of $1.1 \mu \mathrm{m}$ cut-off frequency) and neutral density filters; PD—photodiode (1-reference beam, 2, 3-transmitted beam, 4-reflected beam); $\mathrm{L}_{2}, \mathrm{~L}_{5}$-focusing lenses; $\mathrm{L}_{3}$-collimating lens; $\mathrm{A}$-analyzer.
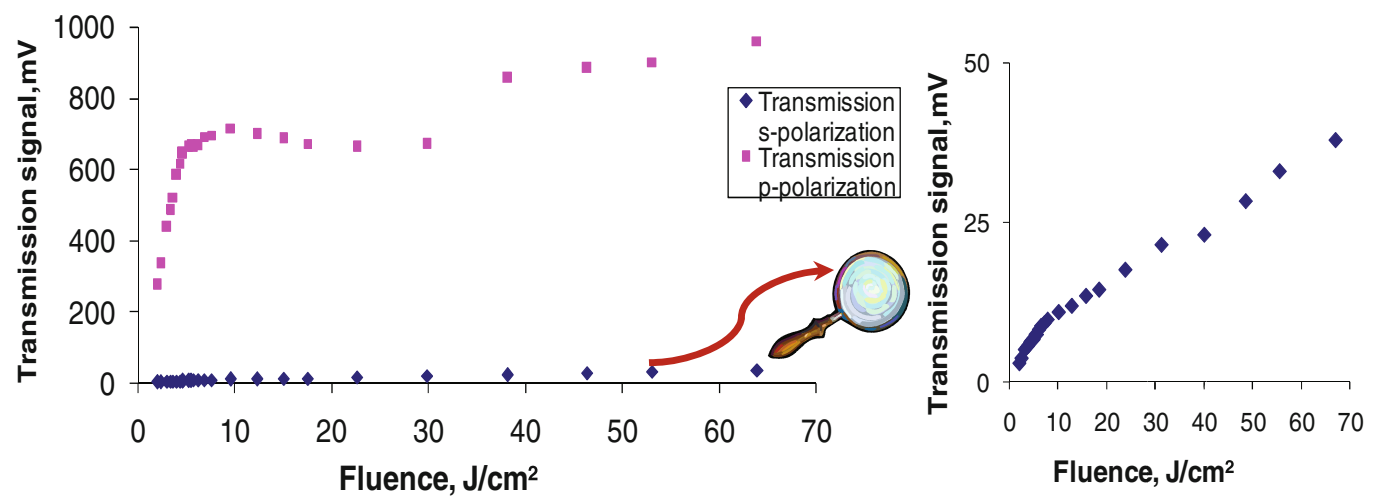

Figure 2. Evolution of the s- and p-polarization components of the transmitted signal versus fluence.

\section{MODELLING}

The model calculates the evolution of reflection, transmission and absorption as a function of time, considering the pulse propogation for a definite fluence. Reflection at the air/ $\mathrm{SiO}_{2}$ surface and transmission (calculated at each layer interface $n_{1}^{*} / n_{2}^{*}$ ) are defined by Fresnel equations using the Drude 
model [1] for the normal incidence:

$$
R_{\|}=\left|\frac{n_{2}^{*}-1}{n_{2}^{*}+1}\right|^{2} ; T_{\|}=\frac{4 n_{1}^{*} n_{2}^{*}}{\left(n_{2}^{*}+n_{1}^{*}\right)^{2}}
$$

Where the refraction index: $n_{i}^{*}=\sqrt{\epsilon_{n e, i}^{*}}=\sqrt{\epsilon_{n e, r, i}^{*}+i \epsilon_{n e, i m, i}^{*}}$ representing the dielectric function $\epsilon_{n e}^{*}$ :

$$
\epsilon_{n e}^{*} \cong 1+\left(\epsilon_{g}-1\right)\left(1-\frac{n_{e}(t, z)}{n_{0}}\right)-\frac{n_{e}(t, z)}{n_{c r}} \frac{1}{1+i \frac{1}{\omega \tau_{c o l}}}
$$

$n_{c r}=\omega^{2} m_{e} \varepsilon_{0} / e^{2}=1.06 \times 10^{21} \mathrm{~cm}^{-3}$ is the critical electron density. The dielectric constant $\varepsilon_{g}=1.963$ for $1025 \mathrm{~nm}$. $e, m_{e}, \epsilon_{0}, \omega$ respectively are the free electron charge, the free electron mass, the vacuum permittivity and the laser pulsation. The initial electron density in the conduction band is taken equal to $n_{0}=2.08 \times 10^{12} \mathrm{~cm}^{-3}$ [2]. $\tau_{c o l}=1 / v_{c o l}$ is the electron-background collision time. The evolution of the electron density $n_{e}(t, z)$ as a function of time and beam propagation inside the material, is calculated considering two major ionization mechanisms: avalanche and photoionization [3]:

$$
\frac{\partial n_{e}(t, z)}{\partial t}=\frac{n_{v}-n_{e}(t, z)}{n_{v}}\left(\sigma_{n} I(t, z)^{n}+\alpha I(t, z) n_{e}\right)-\frac{n_{e}(t, z)}{\tau_{\text {trap }}},
$$

The two terms on the right-hand side represent the free electron generation (multiphotonic ionization term $\sigma_{n} I(t, z)^{n}$ and avalanche term $\left.\alpha I(t, z) n_{e}(t, z)\right)$ and the free electron losses due to the formation of self-trapped excitons, with a trapping time $\tau_{\text {trap }}=150 \mathrm{fs}$ [4]; $I(t, z)$ is the laser intensity; $n_{v}$ is the number of valence electrons. The Keldysh theory [5] is used to compute the cross section of field ionization and yields $\sigma_{n}=\sigma_{8}=1.8 \times 10^{-98} \mathrm{~m}^{5} / \mathrm{sW}^{8}$. The avalanche ionization rate is $\alpha=6 \mathrm{~cm}^{2} / \mathrm{J} \mathrm{[6]}$. We assume that the beam is Gaussian with respect to time: $I(t)=I_{\text {peak }} \exp \left[-\sqrt{\pi} \ln (16)\left(t-t_{0}\right)^{2} / \tau_{0}^{2}\right]$, where $\tau_{0}=\tau / 2 \sqrt{\ln (2)}, I_{\text {peak }}=F / \tau$, the peak laser fluence is defined by $F=2 E / \pi \omega_{0}^{2}$.

\section{RESULTS AND DISCUSSION}

The experimental evolutions of reflection, transmission and absorption are presented in Fig. 3 as a function of laser fluence. The reflection (respectively transmission) increases (resp. decreases) with fluence before saturating for $\mathrm{F} \geq 30 \mathrm{~J} / \mathrm{cm}^{2}$. The onset of $\mathrm{R}$ and $\mathrm{T}$ change roughly corresponds to the damage fluence $\left(4.4 \mathrm{~J} / \mathrm{cm}^{2}\right)$, which is independently determined through a statistical analysis by post-mortem examination of the sample with an optical microscope [7]. At the ablation threshold $\left(6 \mathrm{~J} / \mathrm{cm}^{2}\right.$, as defined by the AFM regression technique [7]), the absorbed energy roughly amounts to $30 \%$ of the incident energy. We then compare the experimental curves to the calculations. The choice

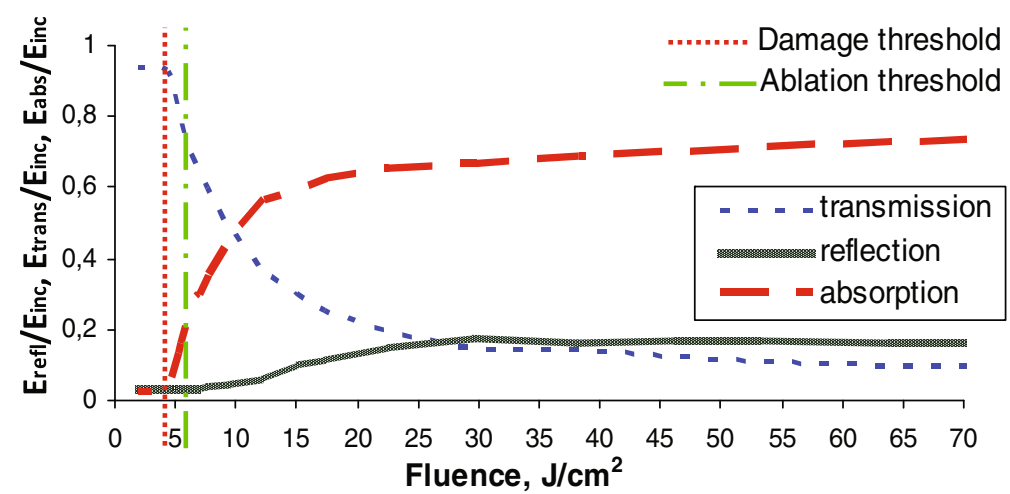

Figure 3. Reflection, Transmission, Absorption of suprasil as a function of fluence. 


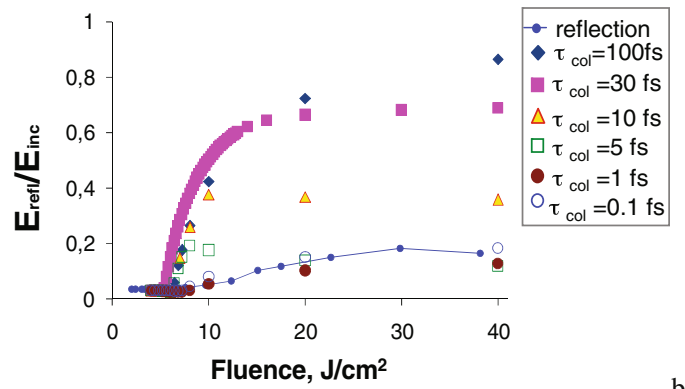

a)

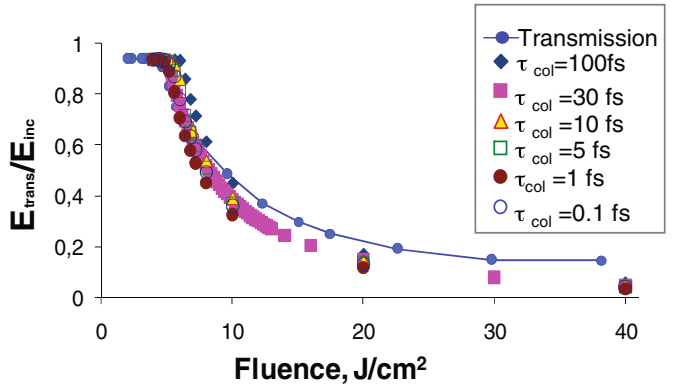

Fluence, $\mathrm{J} / \mathrm{cm}^{2}$

Figure 4. (a)—Reflected energy as a function of fluence; (b) — Transmitted energy as a function of fluence.
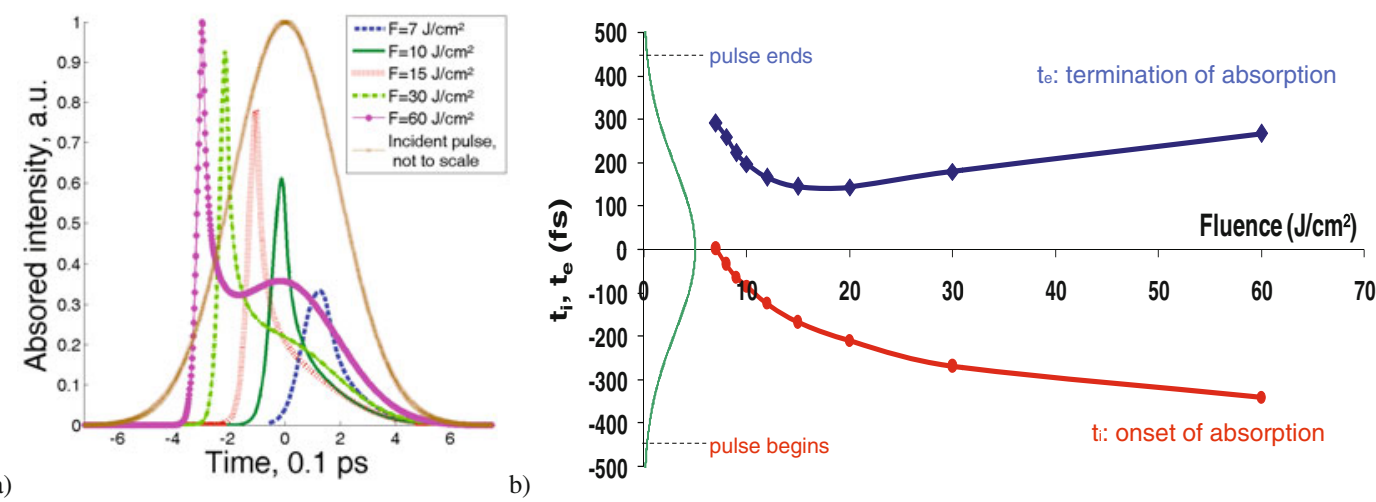

Figure 5. (a) - Theoretical distribution of absorbed energy during femtosecond laser pulse; (b)— Calculation of the beginning $t_{i}$ and end $t_{e}$ of absorption as a function of fluence. The parameteres $t_{i}$ and end $t_{e}$ are calculated at the $1 / e^{2}$ level with respect to the maximal value of absorbed laser energy for each fluence in Fig. 5(a).

of the electron-background collision frequency $v_{c o l}$ is crucial for the modelling [8]. For instance, the reflectivity is very sensible to this parameter. In our model, this parameter was varied in a large range and compared to the experimental results (Fig. 4). The best fit is obtained for a collisional time $\tau_{c o l}=0.1-1$ fs in accordance with [4]. In reality, this parameter is probably variable during the process of energy deposition as the electron density and the electron temperature grow rapidly during the laser pulse. In the following, to understand better the mechanisms of laser pulse absorption as a function of fluence, all the model computations are done for $\tau_{c o l}=0.1 \mathrm{fs}$. Figure 5 illustrates the absorbed energy as a function of fluence and time, yielding important concluding remarks about the dynamics of energy absorption at the surface of the dielectric material. First, the activation of the absorption process is energy (fluence) sensible. Second, the beginning of absorption is moved temporally to the pulse beginning with the increase of the deposited energy. Last, at high fluence $\left(\mathrm{F} \gg 20 \mathrm{~J} / \mathrm{cm}^{2}\right.$ ), we assume that the end of absorption takes place later due to the reionization of trapped excitons.

\section{Acknowledgement}

Financial support of ANR - Nanomorphing-07-BLAN-0301-03 is gratefully acknowledged. 


\section{References}

[1] N.W. Ashcroft, Solid State Physics (Harcourt College Publishers, USA 1976) 2-27

[2] G.M. Petrov, J. Davis, J. of Phys. B: Atom., Molec. and Opt. Phys., 41, (2008) 025601, 1-14

[3] B.C. Stuart, M.D. Feit, S. Herman, A.M. Rubenchik, B.W. Shore, M.D. Perry, Phys. Rev. B 53, (1996) 17491761

[4] P. Martin, S. Guizard, Ph. Daguzan, G. Petite, P. D’Oliveira, P. Meynadier, and M. Perdrix, Phys. Rev. B 55, (1997) 5799-5809

[5] L.V. Keldysh, Zh. Eksp. Teor. Fiz 47, (1964) 1945 (Sov. Phys. JETP, 20, 1307 (1965))

[6] N.M. Bulgakova, R. Stoian, A. Rosenfeld, I.V. Hertel, W. Marine, and E.E.B. Campbell, Appl. Phys. A 81, (2005) 345-356

[7] N. Sanner, O. Utéza, B. Bussiere, G. Coustillier, A. Leray, T. Itina, M. Sentis, Appl. Phys. A 94, (2009) 889-897

[8] D. Arnold, E. Cartier, Phys. Rev. B 46, (1992) 15102-15115 